\title{
To Improve Feature Extraction and Opinion Classification Issues in Customer Product Reviews Utilizing an Efficient Feature Extraction and Classification (EFEC) Algorithm
}

\author{
Palaiyah Solainayagi*1, Ramalingam Ponnusamy ${ }^{2}$ \\ ${ }^{1}$ Research Scholar, Sathyabhama University, Chennai, Tamilnadu, India \\ ${ }^{2}$ Principal, Rajiv Gandhi College of Engineering, Chennai, Tamilnadu, India
}

\section{Article Info}

Article history:

Received Nov 22, 2017

Revised Jan 20, 2018

Accepted Feb 11, 2018

\section{Keywords:}

Classification

Efficient feature extraction and

classification (efec)

Feature extraction

Negative word

Opinion words

Positive word

\begin{abstract}
Currently, customer's product review opinion plays an essential role in deciding the purchasing of the online product. A customer prefers to acquire the opinion of other customers by viewing their opinion during online products' reviews, blogs and social networking sites, etc. The majority of the product reviews including huge words. A few users provide the opinion; it is tough to analysis and understands the meaning of reviews. To improve user fulfillment and shopping experience, it has become a general practice for online sellers to allow their users to review or to communicate opinions of the products that they have sold. The major goal of the paper is to solve feature extraction problem and opinion classification problem from customers utilized product reviews which extract the feature words and opinion words from product reviews. To propose an Efficient Feature Extraction and Classification (EFEC) algorithm is implementing to extracts a feature from opinion words. The reviewer usually marks both positive and negative parts of the reviewed product, despite the fact that their general opinion on the product may be positive or negative. An EFEC algorithm is utilized to predict the number of positive and negative opinion in reviews. Based on Experimental evaluations, proposed algorithm improves accuracy $15.05 \%$, precision $13.7 \%$, recall $15.59 \%$ and F-measure $15.07 \%$ of the proposed system compared than existing methodologies
\end{abstract}

Copyright $\odot 2018$ Institute of Advanced Engineering and Science. All rights reserved.

\section{Corresponding Author:}

Palaiyah Solainayagi,

Department of Computer Science and Engineering,

Sathyabhama University,

Rajiv Gandhi Salai, Jeppiaar Nagar, Chennai, Tamil Nadu 600119.

Email: solaisbu.phd@gmail.com.

\section{INTRODUCTION}

Nowadays, the usage of the Internet enhances at a rapid rate across a wide variety of fields. The rapid development of e-commerce more and more products are bought on the web. Most of the peoples do shopping in online. Not only have the peoples also tended to distribute their experience regarding products on the internet. To improve user fulfillment and shopping experience, it has become a general practice for online sellers to allow their users to review or to communicate opinions of the products that they have sold. Some online users increase the number of reviews conveyed on the internet and also increases at a rapid rate. Some of the products have a lot of reviews. Understanding the user's suggestions about products is very useful for sellers and users who are willing to purchase those products in the future.

Opinion mining is the procedure of mining the opinion word and targets about the specific product. Opinion Mining is described by the task of discovering the opinions of different people concerning different targets. It is interdisciplinary research-oriented phenomenon including fields like natural language 
processing, text mining and analysis, processing and fetching fruitful and subjective data. However, automatic detection and investigation of opinions about products, brands, political issues, and so forth is an overwhelming task. Opinion mining includes three chief components: feature and feature-of relations, opinion expressions and the related opinion attributes (e.g., polarity), and feature-opinion relations. An opinion lexicon is listing of opinion expressions or set of adjectives, which are utilized to demonstrate opinions like positive, negative or neutral. Analyzing all reviews is not efficient when the review amount is huge. Sometimes, the review words are created confusions. The majority of the product reviews including huge words. A few users provide the opinion, and it is tough to analysis and understands the meaning of reviews. The customer review sentences were tagged, opinion words were extracted, and opinion orientations were recognized using the semantic orientation of opinion words. However Positive and negative opinion was identified, but the ranking is not applied to products. The ranking is very useful for users or merchants for buying products. An investigation of online user reviews in which firms cannot determine what accurately people liked and did not like in document-level and sentence-level opinion mining. Hence, nowadays opinion mining research is in phrase-level opinion mining. It performs a fine-grained examination and directly looks at the opinion in online reviews. However, social media sites reporting customer opinions of products in various formats. Monitoring the product opinion is very difficult to find customer reviews.

Shoiab Ahmed et al. [1] designed Sentimental Analysis and Opinion Mining based on SentiWordNet, which produces a count of score words into seven classifications utilizing machine learning algorithms. The web data is gathered utilizing web crawler applied with various preprocessing systems which consist exclusion of stop-words from online reviews, at that point stemming is performed utilizing Porter Stemmer algorithm, and then reviews are labeled utilizing Stanford POS tagger. Rowida Alfrjani et al. [2] introduced a new technique for semantic modeling of the domain knowledge for opinion mining. It focused on modeling the domain knowledge in such way that it can be translated a formal ontology, which would then be able to be automatically improved with ground facts acquired from public Linked Open Data assets.

Nitu Kumari et al. [3] developed the various suggestions to sentiment analysis significantly machine learning sentiment analysis mainly machine learning. E-commerce is not simply purchasing and selling over the Internet; rather it enhances the effectiveness of different rival giants in the market. Opinion mining can contain our product at internet by rating, star system, review of the product. Dhanalakshmi et al. [4] designed Opinion Mining technique for classifying the understudies' feedback obtained during module assessment survey that is conducted each semester to know the feedback of understudies concerning different features of teaching and learning. Opinion mining on the understudy feedback produced through surveys utilizing supervised machine learning algorithms implemented through Rapid Miner. Balahadia et al. [5] developed a teacher's performance assessment tool utilizing opinion mining with sentiment investigation. It gave the sentiment score from the qualitative data and numerical response rating from the quantitative data of teacher's assessment.

Solai Ananth et al. [6] designed the Sentence level Categorizer is utilized for assembling the datasets from Twitter. Datasets area unit was tokenized by TOKENIZER. The tokens area unit was handled by knowledge PREPROCESSING. Naïve Baye's Classifier was classifying the datasets; it is most efficient classifier for Sentence-Level Categorization. Deshmukh et al. [7] developed a bipartite graph clustering was utilized to reduce the mismatch between domain-specific words of source domain and target domain. A domain-independent word was utilized to cluster domain-specific words from a source and target domains. A trained classifier for the target domain, clustering was utilized as it reduced the gap between domain-specific words of various domains. Marrese-Taylor et al. [8] introduced a replicability issues in syntactic centric aspect-based opinion mining. It focused syntactic techniques, which tend to demonstrate a lower level of transparency due to the increasing level of model complexity and the lack of code accessibility. Li et al. [9] described possible directions for deeper understanding, helping bridge the gap between psychology/cognitive science and computational methodologies. It focused on opinion holder's basic needs and their resultant objectives, and functional model of sentiment gave the basis to clarifying the reason a sentiment valence is held. Chandre et al. [10] focused on making a reversed review to help supervised sentiment classification which gives us knowledge in sentiment analysis and opinion mining. Corpus-based pseudo-antonym dictionary implicated a comprehensive, practical approach when compelled with restricted lexical assets and domain knowledge.

Kumar et al. [11] designed to extract the maximum and accurate product features from a huge number of online product reviews. Comprehensive feature extraction approach performed superior to anything the specific way for extracting the product features in the semantic environment. Jain et al. [12] developed a novel localized opinion mining model based on common sense data extracted from the ConceptNet ontology. The technique permitted interpretation and usage of information extracted from webbased social networking sites "Twitter" to identify public opinions. It is utilized to calculate senti-score and build a machine learning model that classifies the client opinions. Arya et al. [13] concentrated on a review of 
Opinion mining and sentiment investigation as it is the process of examining the content (opinion or review) about a subject written in a natural language and characterize them as positive, negative or neutral based on the human's sentiments required in it. Mandal et al. [14] discussed a novel dictionary-based algorithm that utilizations vocabulary-based approach for conclusion mining and computes the assessment extremity levels. Lexicon based approach of content classification for opinion mining was utilized a dictionary containing words that mean feeling. Mane et al. [15] designed a new approach for Opinion Mining of Amazon reviews. The system removed fake reviews and performs opinion mining on genuine reviews to rate the items. The Apache Spark framework was utilized for the speed of processing information expanded and it investigation takes lesser time.

Patel et al. [16] introduced a comparative investigation of various methodologies for multilingual sentiment analysis. These methodologies are partitioned into two sections: one utilizing classification of content without language translation and second utilizing the translation of testing information to a target language before classification. Wu et al. [17] focused on social choice theory and a collective decisionmaking model, it is integrated strategy to help the collective decision-making process based on the analysis of people's social roles and enhanced the effectiveness of the collective decision-making process. Ashok Kumar et al. [18] discussed a multi-aspect based opinion mining framework is proposed for open and distance education to calculate the fulfillment of the public. The framework required for the data gathering process, data preprocessing, feature extraction, opinion discovery at the title level, report level, sentence level, and aspect level, opinion perception, opinion classification. Clavel et al. [19] introduced different avenues for the integration of sentiment analysis in face-to-face human-agent interactions. Sufficient psycholinguistic model utilized to describe human- agent effective dialogues. Semantic rules and machine learning techniques integrated the multi-modal idea of sentiment related phenomena, the variability of temporal and decision frames, differing levels of complexity required by the timing constraint of the interaction, and the heterogeneity. Pham et al. [20] developed a new model based neural network utilizing both known aspect ratings and the overall ratings of reviews to decide the overall aspect weights. The overall rating of a review is derived from aspect ratings. Prakash et al. [22] implemented an approximation automated structure, called Filtered Wall (FW) and it filtered disposed of substance from OSN client substances. The goal is to utilize efficient classification procedure to stay away from overpowered by unsuccessful messages. In OSNs, content filtering can also be abused for a unique, more reactive.

In [23] explained integration of Adaptive Weight Ranking Policy (AWRP) with intelligent classifiers (NB-AWRP-DA and J48-AWRP-DA) via dynamic aging factor to improve classifiers power of prediction. The methods are used to choose the best subset of features. In [24] introduced a new framework called Fuzzy based contextual recommendation system for classification of customer reviews. It extracts the information from the reviews based on the context given by users. In [25] studied to identify the best classifiers for class imbalanced health datasets through a cost-based comparison of classifier performance. The unequal misclassification costs were represented in a cost matrix, and cost-benefit.

To overcome the above problems, proposed an Efficient Feature Extraction and Classification (EFEC) algorithm is designed to solve the opinion classification, feature extraction problem from customer's utilized product reviews which extracts the feature words and opinion words from product reviews dataset. The technique is preferable to utilize the product review reports. Extracted rules are applied on evaluation process to verify whether the rules are appropriate or not. Meantime, the best rules implemented on the preprocessed dataset to extract a feature from opinion words. The reviewer usually marks both positive and negative parts of the reviewed product, despite the fact that their general opinion on the product may be positive or negative. Efficient Feature Extraction and Classification (EFEC) algorithm is utilized to predict the number of positive and negative opinion in reviews. The positive and negative labels gathered in opinion words. Illustrations of review comments are in long sentences. The system extracts from the number of sentences like excellent, good, for positive and poor, bad for negative opinions mining. The subsequent stage is to identify the number of positive and negative opinions of each extracted parts. The paper contributions are given below in details:

- To design an efficient product opinion mining algorithm for finding more accurate product features on opinion mining that have been reviewed on by reviewers.

- To offers robust mechanism for solving product feature extraction problem from customer reviews using Efficient Feature Extraction and Classification (EFEC) algorithm

- $\quad$ To find the number of positive and negative opinion in reviews using opinion words utilizing EFEC methodology.

- $\quad$ To avoiding fake rating reviews from product rating, the effective technique is applied to identify fake reviews online from filter reviews.

- $\quad$ To reduce the product retrieval time, improve product review classification accuracy and success rate compare than existing techniques. 
The rest of papers are organized as: Section 2 express the literature study which is very close to proposed methodology. Section 3 explains the proposed methodology system architecture and implementations pre-processing steps with algorithm pseudo code. Section 4 discusses programming setup, performance evaluation matrix with comparative result analysis. Section 5 summarizes the overall work with future outcomes.

\section{PROPOSED METHOD}

The segment explains the synopsis of the proposed methodology, system architecture, and algorithm explanation with pseudo code. The proposed technique tries to solve complexity for implementation of product feature extraction problem in customer reviews of opinion mining. The feature extraction based opinion mining methods are previously available. However, these methods are unsuccessful in completing the current requirement of opinion mining applications. The here experimental study requires studying to bring the efficient method to complete the gap between current methods and opinion mining application requirements.

\subsection{System Architecture}

In the section established the system architecture with proposed techniques and algorithm details. The system detailed implementation process step by step with design architecture in Figure 1. The objective of the proposed system is finding perfect product features on opinion mining that has been reviewed on by customers. These systems also provide the interaction between customer and administrator is to applying Efficient Feature Extraction and Classification (EFEC) method for solving product feature extraction problem from customer reviews. Proposed Efficient Feature Extraction and Classification (EFEC) algorithm works to classify positive and negative opinions in reviews using opinion words, reduce the product retrieval time, and improve product review classification accuracy and success rate.

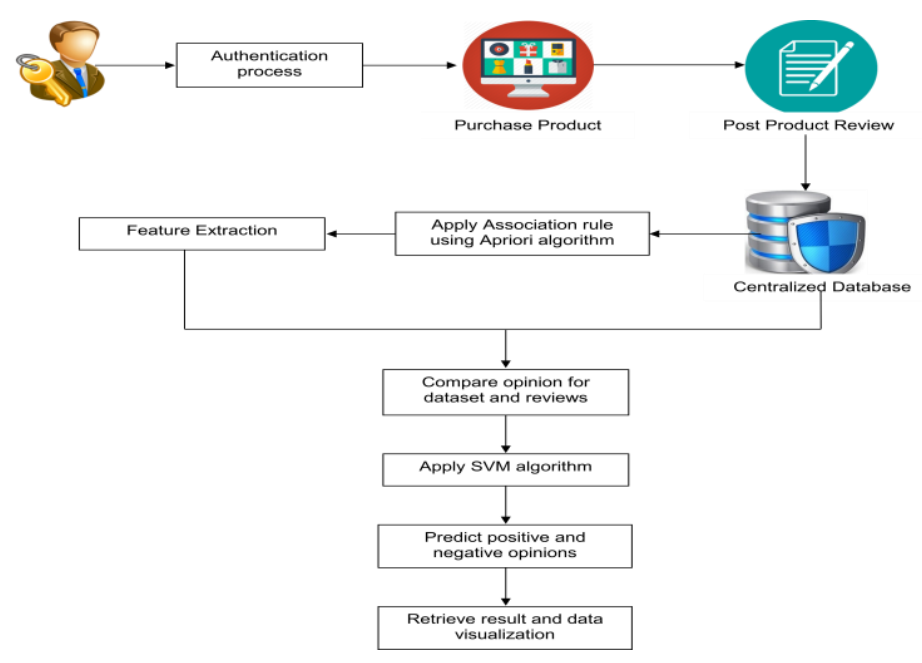

Figure 1. System Architecture Diagram

\subsection{Implementation Pre-processing Steps}

\subsubsection{Authentication}

The customer has to provide the entire details about him/her to generate a new account. After effectively completion of account creation simply the customer can able to perform the online shopping facility. Once the customer submits their details, information is recognized, and the customer can log in with their customer id and password for purchasing products. In the module, it verifies whether the authorized customer is accessing and it does not allow other customers to access.

\subsubsection{Purchasing}

The customer can purchase products and also has the facility to offer reviews with suggestions. The proprietor can contain product features (product name, cost, validity etc.) based on the classification likes mobiles, computers, laptops, cars, etc., and maintain the product details. The customer enters their credit card 
details; the credit card is authenticated. If the card details are legitimate, the customer can purchase their products. The customer can choose purchasing products displayed in the first page or investigate the product utilizing keyword or based on type. The customer can buy the product utilizing credit/debit card. To purchase, the customer requires the following details like (credit card number, cardholder name, date of birth, credit card provider). If the credit card is legitimate, the customer is permitted to purchase the product.

\subsubsection{Feature Extraction}

To Extracting and examine opinions, from product reviews, it is unacceptable to acquire the common opinion about a product simply. In the majority cases, customers expect to discover opinions about feature of a product that is analyzed. Readers hope to realize that the reviewer opinions are positive opinion of the product and a negative opinion of the product, not just the reviewer's general opinion. To satisfying that aim, both product feature and opinion words must be recognized which applying Efficient Feature Extraction and Classification (EFEC) Algorithm. First, however, it is important to extract and build a product feature list and an opinion word lexicon, both of which can give previous knowledge that is helpful for opinion mining.

\subsubsection{Rating}

The customer is permitted to have the facility of giving their review in the form of ratings regarding the service provider. Customer ratings are considered as one of the essential features as they assume a vital part in the purchase of the product. Wrong/unfair ratings may prompt to serious issues in numerous frameworks. So in the module, we collect the customer reviews and secure them.

\subsubsection{Classification}

The entire customer profiles value and reviews are gathered. Customer profiles esteem additionally incorporates their time, duration and reviews and so forth. All the customer profiles including evaluations esteems are saved safely. Every one of the information's gathered is utilized as a dataset. In the Dataset, we classify the Positive and Negative opinions by amount of reviews provided using SVM algorithm.

\subsubsection{Positive and Negative Reviews}

In the module, develop the framework to such that customer of the portal can have the rights to give the positive and negative reviews to the product which he/she purchases, such that the administrator can view the list of reviews.

\subsection{Efficient Feature Extraction and Classification (EFEC) Algorithm}

To discovering the various product features, EFEC algorithm is utilized to enhance feature extraction and classification of positive and negative opinions. In the framework, a product set is a set of words or a phrase that occurs together. The method predicts features that appear on a lot of opinions have more possibility to be related, and consequently, more likely to be a real product feature. The classification algorithm is utilized to find the amount of positive, negative or neutral opinion words in product reviews utilizing opinion words. Opinion words are encoded a big situation like excellent, good have a positive opinion, while opinion words that stand for unwanted states like poor, bad, disappointing have a negative opinion. The word includes opinion words are taken as opinion sentence. Illustrations of positive opinion words are excellent and good, and the negative opinion words are like poor, bad and so on. To recognize the amount of positive, negative or neutral product opinions of every extracted product feature. These opinion words are extracted utilizing Efficient Feature Extraction and Classification (EFEC).

An Efficient Feature Extraction and Classification is a classification algorithm utilized for text classification. The product review text to be categorized is changed into word. EFEC builds a hyper plane utilizing these words which divides data illustrations of one class from another. The unique feature of EFEC is that it can study. Still, it gives large data. It efforts glowing for text classification since it can handle huge features. Improvement of EFEC is robust when there is a small set of illustrations allocated over a huge region. It has provided reliable outcomes in the research in opinion mining. A product review data categorization is a two-stage procedure. In the first stage, a categorization algorithm constructs the classifier by "learning from" a training set made up of our corpus and their correlated class labels. In the second stage, the model is used for categorization. A different set called test set is utilized to estimate the exactness of the constructed model. 
Input: Load the input Dataset DB (Customer Review Datasets)

Output: Visualize the Tabular Result TR with Quality of Information (Accuracy, Precision, Recall and F-measure)

Begin:

User authentication process

Purchase any product

Post the feedback on product reviews

Store centralized database

Apply EFEC algorithm

Collection of opinion word in container of keywords

Extract the features

If feature is positive

To predict positive features

Else

To predict negative features

End If

Compare opinions from feature dataset and reviews

If negative word appears in feature

Negative opinion word is predicted

Else

Positive opinion word is predicted

End If

Predict positive and negative opinions

Retrieve result and data visualization

Calculate accuracy, precision, recall and F-measure

End.

\section{RESULTS AND ANALYSIS}

\subsection{Programming Setup}

To comparing, the proposed system with existing methodologies, the deployment procedure is performed on a system among Intel Core i7 7600 processor, 8GB memory, along with Window 7 system. Here, the method implemented in JAVA using NetBeans 8.0 with Apache Tomcat 8.0.3 and MYSQL 5.5 Database. The Proposed algorithm is calculated with numerous kinds of opinion dataset to estimate the effectiveness of proposed systems.

\subsection{Dataset}

The proposed methodology utilized Customer Review Datasets (CRD) which includes reviews about product efficiently. A product review is a personal text including a sequence of words defining opinions of reviewer considering a particular product. Product review text may include complete sentences, little remarks, or both. Product reviews are gathered from websites like www.amazon.com, www.epinions.com and www.cnet.com. Every product review in websites is allotted with a different rating like 0-5 stars, a product review date, a reviewer name and location, a manufactured products name, and the product review content.

\subsection{Performance Evaluation Matrix}

The proposed methodology discovers the estimation metrics namely accuracy, precision, recall and F-measure to compute effectiveness of the proposed method and overcome the previous mechanisms in opinion mining. In the methodology enhances feature extraction and opinion classification. The methodology calculates the accuracy, precision, recall and F-measure. The following evaluation parameters are explained below in details.

\subsubsection{Accuracy}

Accuracy is defined as the sum of the true predictions such as divided by the total number of predictions. True positives and true negatives are described as the number of products correctly estimated as positive and negative. False positives and false negatives are defined as the number of products incorrectly computed as positive and negative. 


$$
\text { Accuracy }=\frac{\text { True Positives }+ \text { True Negatives }}{\text { True Positives }+ \text { True Negatives }+ \text { False Positives }+ \text { False Negatives }}
$$

\subsubsection{Precision}

Precision is described as the ratio of products is correctly predicted positives divided by total products are correctly and incorrectly predicted positives.

$$
\text { Precision }=\frac{\text { True Positives }}{\text { True Positives }+ \text { False Positives }}
$$

\subsubsection{Recall}

The recall is defined as the ratio of products correctly predicted as positives divided by the sum of products is correctly predicted as positives and sentences are incorrectly predicted as negatives.

$$
\text { Recall }=\frac{\text { True Positives }}{\text { True Positives }+ \text { False Negatives }}
$$

\subsubsection{F-measure}

F-measure is described as the weighted harmonic mean of precision and recall. The F1 measure communicates the balance between the precision and the recall.

$$
F-\text { measure }=\frac{2 * \text { Precision } * \text { Recall }}{\text { Precision }+ \text { Recall }}
$$

Table 1. Comparison of Accuracy, Precision, Recall, and F-measure

\begin{tabular}{ccccc}
\hline Algorithm & Accuracy & Precision & Recall & F-measure \\
\hline Naïve Bayes & 74.76 & 79.54 & 75.86 & 73.75 \\
SVM & 82.85 & 84.45 & 82.13 & 82.38 \\
ME & 79.04 & 81.75 & 79.99 & 78.59 \\
EFES+J48 & 97.90 & 98.15 & 97.72 & 97.45 \\
\hline
\end{tabular}

Table 1 demonstrates the Accuracy, Precision, Recall and F-measure for input aspects with existing methodologies. Table 1 shows the average value of all estimation aspects with input aspects. The proposed system is estimated with following existing classifiers namely: Naïve Bayes [21], Support Vector Machine (SVM) [21] and Maximum Entropy [21] classifiers. According to Table1, it noticed that EFEC algorithm has the best score on every specify aspects for classification.

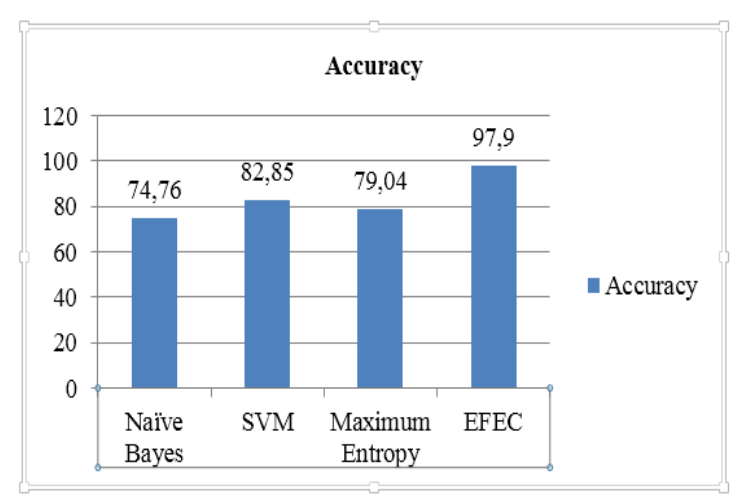

Figure 2. Comparison of Accuracy with Various Algorithms

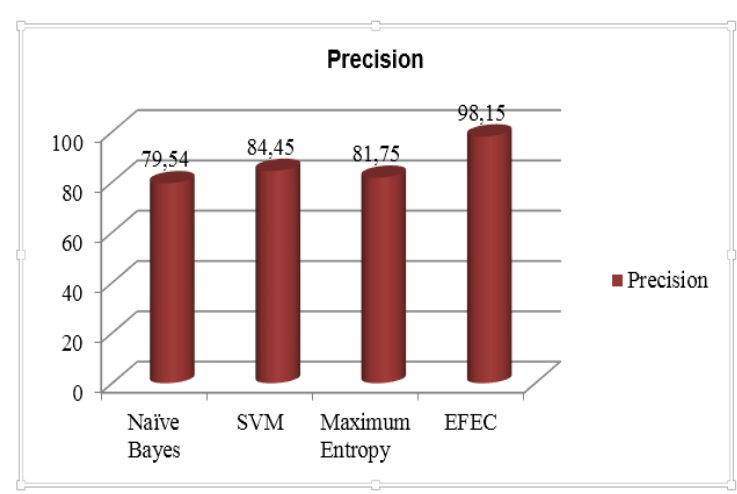

Figure 3. Comparison of Precision with Various Algorithms 


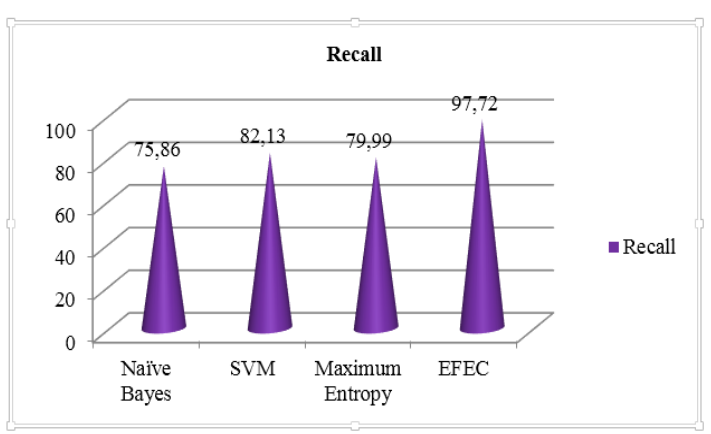

Figure 4. Comparison of Recall with Various Algorithms

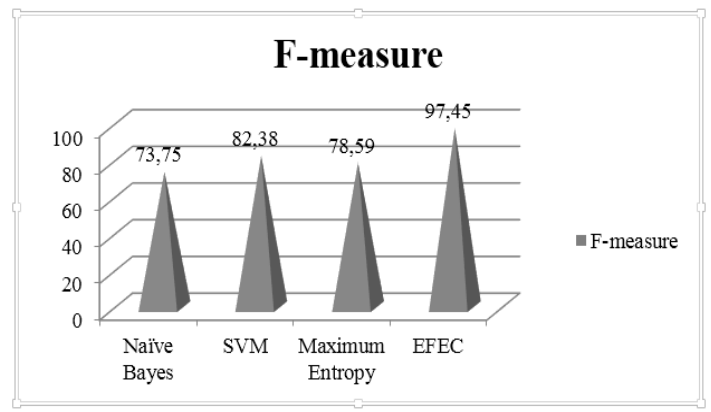

Figure 5. Comparison of F-measure with Various Algorithms

According to Figure 2 to 5 estimations, it observed that the proposed technique is evaluated based on accuracy, precision, recall, and F-measure. Proposed EFEC is estimated with Naïve Bayes (NB) [21], Support Vector Machine (SVM) [21] and Maximum Entropy (ME) [21] methodologies behalf of accuracy, precision, recall and F-measure. SVM is the closest challenger. It enhances the classification problem of product reviews. However, SVM is provided with the less accuracy. An EFEC strategy enhances the feature extraction and classification accuracy $15.05 \%$, precision $13.7 \%$, recall $15.59 \%$ and F-measure $15.07 \%$. Lastly, the paper declares the proposed EFEC algorithm is best in all several aspects).

\section{CONCLUSION}

In Conclusion of the paper, a proposed technique extract features in product reviews. The nouns and noun phrases are extracted from every product review. An Efficient Feature Extraction and Classification (EFEC) methodology is utilized to find all various features for the provided product review. Efficient Feature Extraction and Classification (EFEC) method is utilized to recognize whether the sentence is positive or negative opinion and also recognize the amount of positive and negative opinion of every extracted feature. The amount of positive and negative opinions in a product review is calculated. An opinion word provides the classification accuracy. An EFEC strategy enhances the feature extraction and classification accuracy $15.05 \%$, precision $13.7 \%$, recall $15.59 \%$ and F-measure $15.07 \%$. Finally, the paper declares the proposed EFEC algorithm is best in all several aspects.

In future, the paper can be extended to extract the trustworthy user opinion to improve the product quality and user interaction behalf of product from prior user experience in Hadoop Environment.

\section{REFERENCES}

[1] Ahmed, S., Danti, A., "A novel approach for Sentimental Analysis and Opinion Mining based on SentiWordNet using web data", In Trends in Automation, Communications and Computing Technology (I-TACT-15), 2015 International Conference, vol.1, pp.1-5, 2015.

[2] Alfrjani, R., Osman, T., Cosma, G., "A new approach to ontology-based semantic modelling for opinion mining", In Computer Modelling and Simulation (UKSim), 2016 UKSim-AMSS 18th International Conference, 2016, pp. 267-272.

[3] Kumari, N., Singh, S. N., "Sentiment analysis on E-commerce application by using opinion mining”. In Cloud System and Big Data Engineering (Confluence), 2016 6th International Conference, 2016, pp.320-325.

[4] Dhanalakshmi, V., Bino, D., Saravanan, A. M., "Opinion mining from student feedback data using supervised learning algorithms”, In Big Data and Smart City (ICBDSC), 2016 3rd MEC International Conference, 2016, pp. 1-5.

[5] Balahadia, F. F., Fernando, M. C. G., Juanatas, I. C., "Teacher's performance evaluation tool using opinion mining with sentiment analysis”, In Region 10 Symposium (TENSYMP), pp. 95-98, 2016.

[6] Solai Ananth, S. S., Chandu, P. M. S. S., Scholar, P. G., "Live Twitter Knowledge as a Corpus for Sentiment Analysis and Opinion Mining”, International Journal of Engineering Science, vol.7, no.1, pp. 4055-4058, 2017.

[7] Deshmukh, J. S., Tripathy, A. K.., "An entropy-based classifier for cross-domain opinion mining", Applied Computing and Informatics, vol. 14, pp. 55-64, 2017. 
[8] Marrese-Taylor, E., Matsuo, Y., "Replication issues in syntax-based aspect extraction for opinion mining", arXiv preprint arXiv:1701.01565, pp. 1-10, 2017.

[9] Li, J., Hovy, E., "Reflections on sentiment/opinion analysis", In A Practical Guide to Sentiment Analysis, Springer International Publishing, pp. 41-59, 2017.

[10] Chandre, P. R., Raj, R. R. H., "New Avenues in opinion mining: Considering Dual Sentiment Analysis", International Journal of Scientific Research and Education, vol. 5, no.3, pp. 6266- 6273, 2017.

[11] Kumar, K. R., Santosh, D. T., Vardhan, B. V., "Extracting Opinion Targets from Product Reviews using Comprehensive Feature Extraction Model in Opinion Mining”, Indian Journal of Science and Technology, vol.10, no. 21, pp.1-6, 2017.

[12] Jain, A., Jain, M., "Location-based Twitter Opinion Mining using Common-Sense Information", Global Journal of Enterprise Information System, vol. 9, no.2, pp. 28-32, 2017.

[13] Arya, P., mit Bhagat, A., MANIT, B., "Deep Survey on Sentiment Analysis and Opinion Mining on Social Networking Sites, E-Commerce Website”,. International Journal of Engineering Science, vol. 7, no.3, pp. 47964810, 2017.

[14] Mandal, S., Gupta, S., "A novel dictionary-based classification algorithm for opinion mining”, In Research in Computational Intelligence and Communication Networks (ICRCICN), 2016 Second International Conference, 2016, pp. 175-180.

[15] Mane, S. B., Assar, K., Sawant, P., Shinde, M., "Product Rating using Opinion Mining", International Journal of Computer Engineering In Research Trends, vol. 4, no. 5, pp.161-168, 2017.

[16] Patel, S., Nolan, B., Hofmann, M., Owende, P., Patel, K., "Sentiment Analysis: Comparative Analysis of Multilingual Sentiment and Opinion Classification Techniques", World Academy of Science, Engineering and Technology, International Journal of Computer, Electrical, Automation, Control and Information Engineering, vol. 11, no. 6, pp. 565-571, 2017.

[17] Wu, B., Zhou, X., Jin, Q., Lin, F., Leung, H., "Analyzing social roles based on a hierarchical model and data mining for collective decision-making support", IEEE Systems Journal, pp. 1-10, 2015.

[18] Kumar, A., Campus, C. E. G., Murugappan, A., "A Multi-Aspect Based Opinion Mining System for Open and Distance Education Using Online Reviews”, The Online Journal of Distance Education and e-Learning, vol. 5, no.1, pp. 1-11, 2017.

[19] Clavel, C., Callejas, Z., "Sentiment analysis: from opinion mining to human-agent interaction", IEEE Transactions on affective computing, vol. 7, no. 1, pp. 74-93, 2016.

[20] Pham, D. H., Le, A. C., "A Neural Network based Model for Determining Overall Aspect Weights in Opinion Mining and Sentiment Analysis", Indian Journal of Science and Technology, vol. 9, no. 18, pp. 1-6, 2016.

[21] Pujari, C., Shetty, N. P., "Comparison of Classification Techniques for Feature-Oriented Sentiment Analysis of Product Review Data”, In Data Engineering and Intelligent Computing. Springer, Singapore, pp. 149-158, 2018.

[22] Prakash, G., Saurav, N., Kethu, V. R., "An Effective Undesired Content Filtration and Predictions Framework in Online Social Network", International Journal of Advances in Signal and Image Sciences, vol. 2, no. 2, pp. 1-8, 2016.

[23] Olanrewaju, R. F., Azman, A. W., "Intelligent Cooperative Adaptive Weight Ranking Policy via dynamic aging based on NB and J48 classifiers", Indonesian Journal of Electrical Engineering and Informatics (IJEEI), vol. 5, no. 4, pp. 357-365, 2017.

[24] Sulthana, R., Ramasamy, S., "Context Based Classification of Reviews Using Association Rule Mining, Fuzzy Logics and Ontology", Bulletin of Electrical Engineering and Informatics(BEEI), vol. 6, no.3, pp. 250-255, 2017.

[25] Rao, R. R., Makkithaya, K., "Learning from a Class Imbalanced Public Health Dataset: a Cost-based Comparison of Classifier Performance", International Journal of Electrical and Computer Engineering(IJECE), vol. 7, no. 4, pp. 2215-2222, 2017. 